\title{
Role of early genes in pathogenesis of adenovirus pneumonia
}

\author{
(adenovirus DNA-binding protein/adenovirus DNA polymerase/adenovirus late gene products/fiber protein)
}

\author{
Harold S. Ginsberg*, Robert L. Horswood ${ }^{\dagger}$, Robert M. Chanock ${ }^{\dagger}$, and Gregory A. Prince $\ddagger$ \\ ${ }^{*}$ Departments of Medicine and Microbiology, Columbia University College of Physicians and Surgeons, New York, NY 10032; ${ }^{\dagger}$ Laboratory of Infectious \\ Diseases, National Institute of Allergy and Infectious Diseases, Bethesda, MD 20892; and $\ddagger$ Department of International Health, School of Hygiene \\ and Public Health, Johns Hopkins University, Baltimore, MD 21205
}

Contributed by Harold S. Ginsberg, May 29, 1990

\begin{abstract}
Intranasal inoculation of type 5 adenovirus into the cotton rat Sigmodon hispidus produces a pneumonia pathologically similar to that in humans, and it, therefore, provides an excellent animal model to investigate the pathogenesis of this disease. The goal of this study was to test the hypothesis that accumulation of viral structural proteins is responsible for a major portion of the cell-damage-producing disease. Since viral DNA replication is essential for synthesis of the viral structural proteins, which are products of late genes, the hypothesis was tested using mutants defective in genes required for DNA synthesis. Most experiments were done with the conditionally lethal temperature-sensitive (ts) mutant H5ts125, which contains a mutation in the early region $2 \mathrm{~A}$ $(E 2 A)$ gene encoding the DNA-binding protein. The data show that infection with $1 \times 10^{9.0}$ plaque-forming units of H5ts125 induced a pneumonia that was as extensive and qualitatively the same as that after wild-type adenovirus type 5 infection, although H5ts125 did not replicate to produce infectious virus. When cotton rats were infected with $1 \times 10^{8.0}$ plaque-forming units of wild-type adenovirus type 5 or H5ts125, the pneumonias that followed were pathologically similar; in the latter phases, however, wild-type virus produced slightly more extensive pneumonia than did H5ts125, probably because its replication permitted infection of more susceptible cells.
\end{abstract}

The discovery of adenoviruses $(1,2)$, an important human pathogen whose 42 viral types produce a variety of diseases, led to extensive studies into the virion structure, the viral structural proteins, the nature of the viral genome, and the biochemical and molecular events in the sequential biosynthesis of the viral gene products (3). During type 5 adenovirus (Ad5) replication, host cell DNA synthesis is completely blocked about $12 \mathrm{hr}$ after infection with $\operatorname{Ad5}(4,5)$, and host RNA and protein syntheses are blocked about 18-20 hr after infection (5-7). In addition, only about $10 \%$ of the viral DNA and structural proteins synthesized are actually packaged into mature virions whereas the remainder accumulate in the nuclei of infected cells forming inclusion bodies (8-11). These findings led to the hypothesis that the combination of shut-off of biosynthesis of host macromolecules and the intranuclear accumulation of large amounts of viral DNA and structural proteins leads to cell death and production of disease (5). Indeed, the purified fiber protein inhibited viral multiplication when added to adenovirus-infected KB cells, and it was shown to inhibit incorporation of $\left[{ }^{3} \mathrm{H}\right]$ thymidine into cellular DNA in cell extracts in vitro $(12,13)$.

The objective of the study to be described was to test the hypothesis that production of an excess of late viral proteins is responsible for the molecular events essential for the pathogenesis of adenovirus disease. Thus, if synthesis of the late structural proteins were blocked, disease should not

The publication costs of this article were defrayed in part by page charge payment. This article must therefore be hereby marked "advertisement" in accordance with 18 U.S.C. $\$ 1734$ solely to indicate this fact. develop. This possibility was readily tested since mutants unable to synthesize viral DNA cannot produce late structural proteins, and such mutants had been isolated (14). H5ts125 (14) and H5ts149 (15) are conditionally lethal temperature-sensitive (ts) mutants that cannot replicate their DNA at their nonpermissive temperature of $39.5^{\circ} \mathrm{C}$, which fortunately is close to the body temperature of the experimental animal to be employed, the cotton rat $(16,17)$.

\section{MATERIALS AND METHODS}

Cell Cultures. As described $(14,17)$, monolayer cultures of KB and A549 cells were propagated in Dulbecco's modified Eagle's medium supplemented with $10 \%$ (vol/vol) calf serum.

Viruses and Assay Methods. Wild-type (wt) Ad5 and two ts mutants, H5ts125 and H5ts149 $(14,15)$, were used in these studies. Stocks of wt virus were replicated in KB cells at $37^{\circ} \mathrm{C}$; $\mathrm{H} 5$ ts 125 and $\mathrm{H} 5$ ts 149 were propagated at $32^{\circ} \mathrm{C}$, the permissive temperature. Viral infectivities of infected cotton rat homogenates were determined in $\mathrm{KB}$ cells with an indirect immunofluorescence assay at $32^{\circ} \mathrm{C}$ using polyclonal rabbit antiserum prepared with intact purified virus (18). Stock viruses were quantitated using plaque assays (19) in A549 cells.

Animal Studies. An inbred strain of cotton rats, Sigmodon hispidus, 3-6 weeks of age were employed as described (16, 17). The animals were obtained from Veterinary Resources Branch, Division of Research Services, National Institutes of Health. In each experiment animals of the same age were used. Animals were infected intranasally with $0.1 \mathrm{ml}$ of diluted virus while under light inhalation methoxyflurane anesthesia. At the prescribed times of sacrifice, lungs were prepared for histologic examination and viral infectivity assays (refs. 15 and 17 and unpublished results).

Semiquantitative Scoring of Lung Pathology. To compare the extent of the pneumonia in cotton rats infected with different viruses and examined at different intervals after infection, histological sections were examined without identification of virus inoculated or interval after infection (i.e., they were scored "blind"). The number of lobes involved, the degree of bronchiolar epithelial cell damage, and the extent of alveolar, peribronchial, and perivascular inflammatory responses were graded on a scale of 0-4. Data are expressed as mean scores of four to six animals.

\section{RESULTS}

Replication of Ad5 wt and H5ts125 in Cotton Rat Lungs. To determine whether late viral gene products (i.e., viral structural proteins and, thus, viral replication) are essential to produce adenovirus pneumonia in the cotton rat model, Ad5

Abbreviations: Ad5, type 5 adenovirus; wt, wild type; ts, temperature sensitive; TNF, tumor necrosis factor; pfu, plaque-forming unit(s); PMN, polymorphonuclear leukocyte. 
ts mutants unable to replicate their DNA at the nonpermissive temperature were selected, since late structural proteins cannot be produced if viral DNA is not synthesized (20). The conditionally lethal, ts mutants chosen for these experiments have defects in the gene encoding either the DNA-binding protein or the viral DNA polymerase, located in early regions $E 2 A$ and $E 2 B$, respectively $(14,15,21)$. In cell cultures these mutants cannot replicate at $39.5^{\circ} \mathrm{C}$ and, if these restrictions prevail in cotton rats, whose body temperature is 39.0 $39.2^{\circ} \mathrm{C}$, then these viruses also should not be able to replicate their DNAs in the infected lung epithelial cells. Fig. 1 graphically presents results of typical experiments comparing the multiplication of Ad5 wt virus and $\mathrm{H} 5 \mathrm{ts} 125$ after intranasal infection with either $1 \times 10^{8}$ or $1 \times 10^{9}$ plaque-forming units (pfu). Even replication of Ad5 wt virus was limited, and, when $1 \times 10^{9.0} \mathrm{pfu}$ was used, multiplication was undetectable because of the high background from the large inoculum. These data clearly show, however, that H5ts125 cannot produce infectious virus in the lungs of cotton rats any more efficiently than in susceptible cells in culture at the nonpermissive temperature and, thus, that only early gene functions can be expressed under these conditions (20). Experiments using H5ts149 yielded similar results (data not presented). It is important to note that both mutants replicated like wt virus in epithelial cells of the nares (data not shown) where the temperature is about $32^{\circ} \mathrm{C}$, the mutants' permissive temperature (14).

Pneumonias Produced by Ad5 wt and H5ts125. Since H5ts125 did not replicate to produce new virions in epithelial cells of cotton rat lungs because of the restrictive body temperature, it was desirable to use a large viral inoculum to permit expression of early gene functions in an optimum number of bronchial epithelial cells in which wt virus normally replicates (ref. 17 and unpublished results). Cotton rats were inoculated intranasally with $1 \times 10^{9}$ pfu of either Ad5 wt or H5ts125; at intervals after infection viral infectivities were assayed, and the development of pneumonia was examined histologically. Fig. 2 presents pictures of typical pathological responses to both viruses. It is noteworthy that the responses to infection with wt virus and $\mathrm{H} 5$ ts 125 were similar relative to the characteristics of the alveolar (Fig. $2 A$ and $C$ ), peribronchial (Fig. $2 B$ and $D$ ), and perivascular infiltrations.

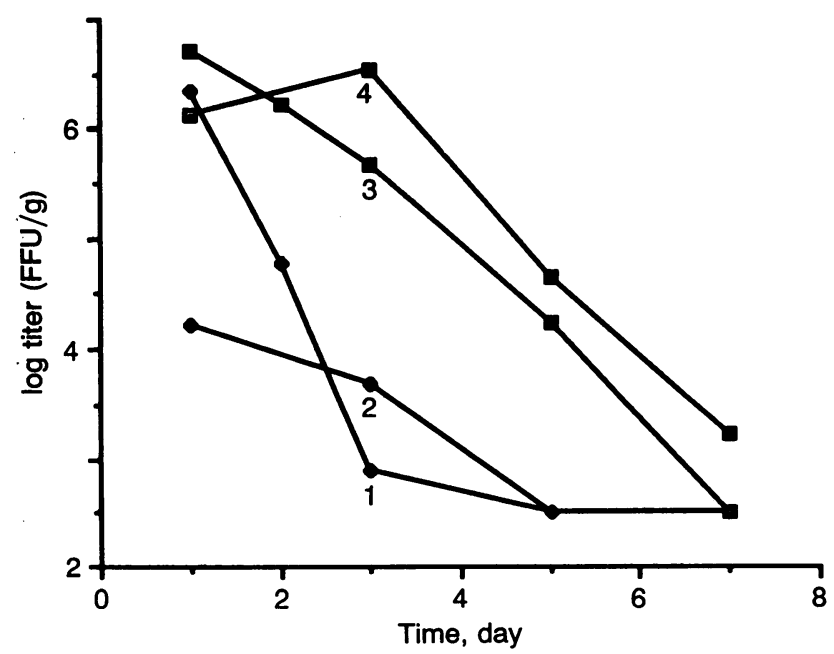

FIG. 1. Multiplication of Ad5 wt and H5ts125 in lungs of cotton rats. Animals were infected intranasally with either $1 \times 10^{9.0}$ or $1 \times$ $10^{8.0} \mathrm{pfu}$. Animals were sacrificed at indicated times after infection, infectivity titers were assayed by fluorescent-focus assays on $10 \%$ lung suspensions (by weight), and geometric mean titers of six animals per group were calculated and plotted. FFU, fluorescentfocus unit(s). Curves: $1,1 \times 10^{9.0} \mathrm{H} 5 \mathrm{st125} ; 2,1 \times 10^{8.0} \mathrm{H} 5 \mathrm{ts} 125 ; 3$, $1 \times 10^{9.0}$ Ad5 wt; $4,1 \times 10^{8.0}$ Ad5 wt.
To substantiate the similarity of the pathological responses to both viruses, all lungs were scored using a semiquantitative method (17) in which the extent of the pathological lesions was scored "blindly". The results of a representative experiment are summarized in Fig. 3. These data confirm the qualitative findings (Fig. 2) that since H5ts125 cannot produce infectious virus in the cotton rat lung, because of its inability to replicate its DNA at its nonpermissive temperature in the lung, only the viral early gene functions could be responsible for the pathogenic process.

Infection of cotton rats with 10 times less H5ts125 (i.e., 1 $\times 10^{8} \mathrm{pfu}$ ) also produced a pneumonia that was pathologically like that effected by Ad5 wt virus. However, during the latter phases of the pathogenesis the infiltration was slightly, but significantly, greater in lungs of animals receiving wt virus although qualitatively the pathology did not differ. Since Ad5 wt virus multiplied in the bronchial epithelial cells and, therefore, could spread and infect more cells than accomplished with the initial inoculum of nonreplicating H5ts125, it was anticipated that the pneumonia would be somewhat more extensive with wt virus. The quantitative similarity of the pneumonias produced by Ad5 wt and H5ts125 viruses when $1 \times 10^{9}$ pfu were used for infection supports this interpretation.

H5ts 149 is a conditionally lethal ts mutant unable to replicate its DNA at $39.5^{\circ} \mathrm{C}$ because of a mutation in its DNA polymerase gene $(15,21)$. Therefore, H5ts 149 , like H5ts 125 , was defective in viral replication at the body temperature of the cotton rat. After intranasal inoculation, H5ts149 also produced pneumonia that was qualitatively similar and quantitatively almost the same as that effected by Ad5 wt (data not presented).

\section{DISCUSSION}

Data obtained from the studies summarized above clearly indicate that viral early gene functions provide the molecular mechanisms that induce adenovirus pneumonia, at least in the cotton rat experimental model (ref. 17 and unpublished results). This finding refutes our earlier hypothesis that the extensive accumulation of unpackaged viral structural proteins, particularly fibers and hexons, produces injury and death of infected epithelial cells and, thus, induction of adenovirus disease (5). Clearly, accumulation of excess structural proteins and virions form the diagnostic intranuclear inclusion bodies present in productively infected cells in human diseases as well as in the cotton rat model of pneumonia (unpublished results). However, H5ts142, a mutant defective in its gene encoding the fiber protein, and thus unable to synthesize fiber and assembly virions $(14,22)$, also effected a pneumonia to the same extent as wt virus (unpublished data). Although other late proteins are made in H5ts142-infected cells, these findings are consistent with the conclusion that early gene functions are responsible for adenovirus pneumonia in the cotton rat. The early gene(s) responsible have not yet been clearly identified, but mutants containing defects in each of the early regions should be tested to determine which early gene functions are necessary to produce disease in the cotton rat model. For example, the early region $1 \mathrm{~B}(E 1 B)$ gene encoding the $58-\mathrm{kDa}$ protein appears to be involved: The mutant $\mathrm{H} 5 \mathrm{~d} 1110$, which contains a large deletion between base pairs 2333 and 2804 (23), multiplies almost as well as Ad5 wt in cotton rat lungs but produces a significantly less extensive pneumonia (unpublished data). The E1B 58-kDa protein is critical for shutting off host protein synthesis in adenovirus-infected cells (23, 24 ), and the failure of $\mathrm{H} 5 \mathrm{dl} 110$ to produce as severe a pneumonia as Ad5 wt virus suggested that the shut-off of host cell protein synthesis is a critical process in the induction of adenovirus pneumonia. 
A

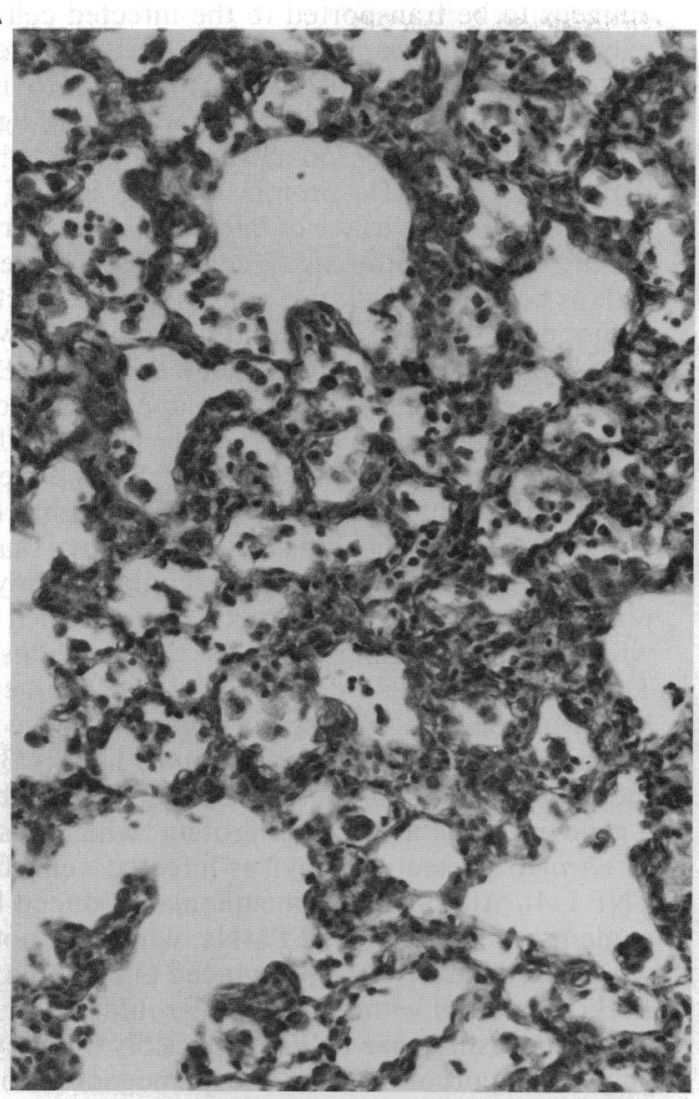

C

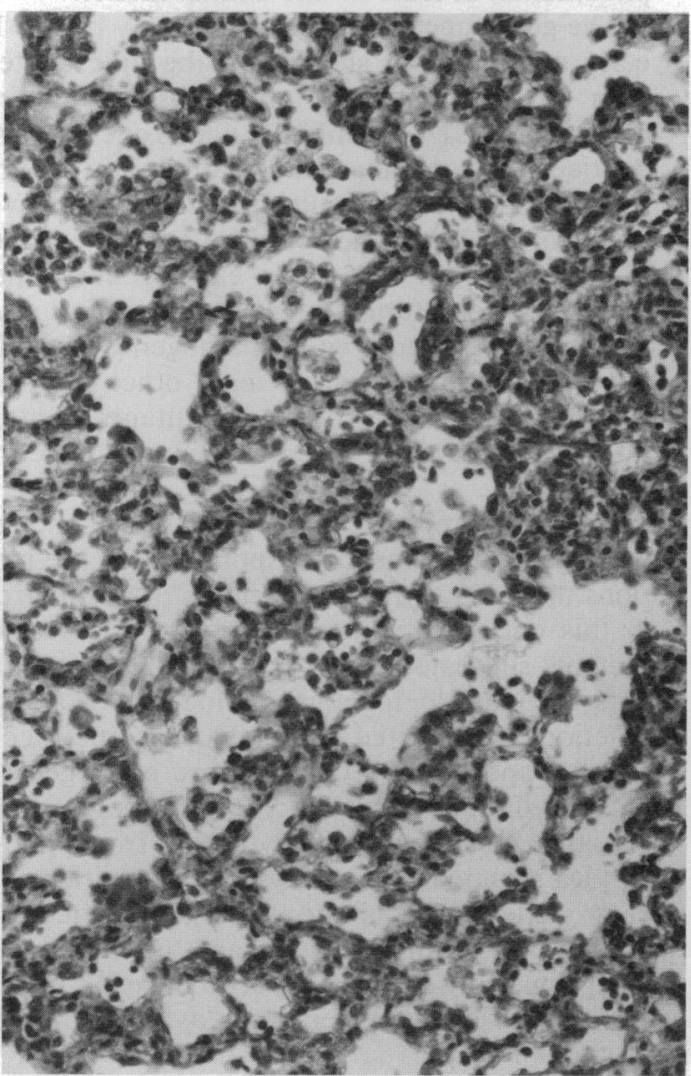

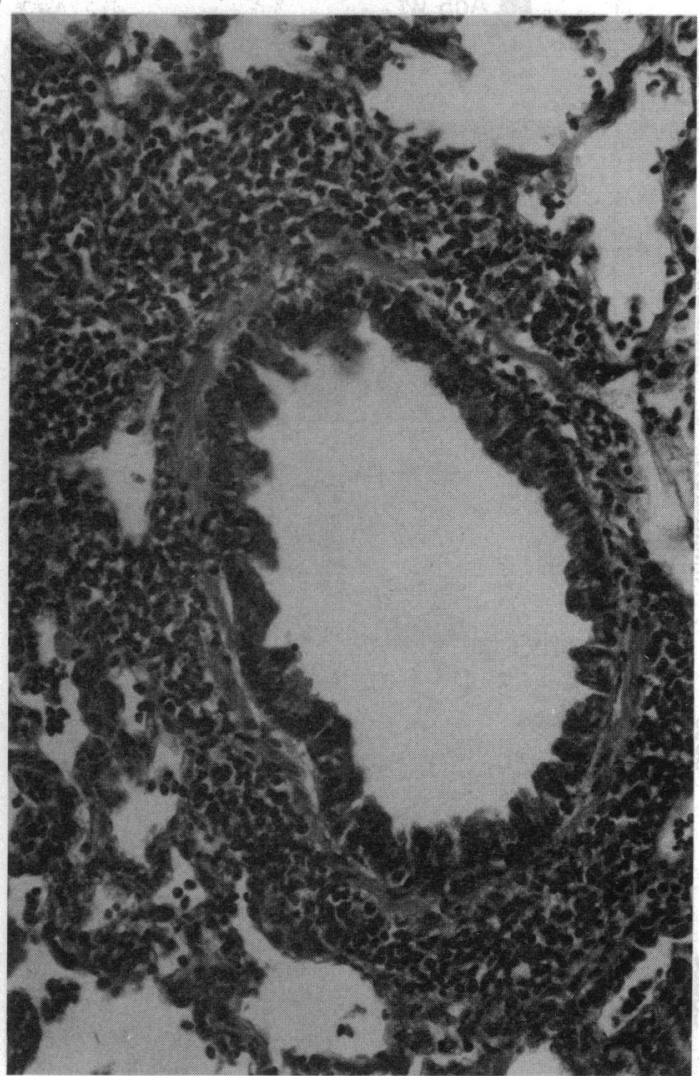

D

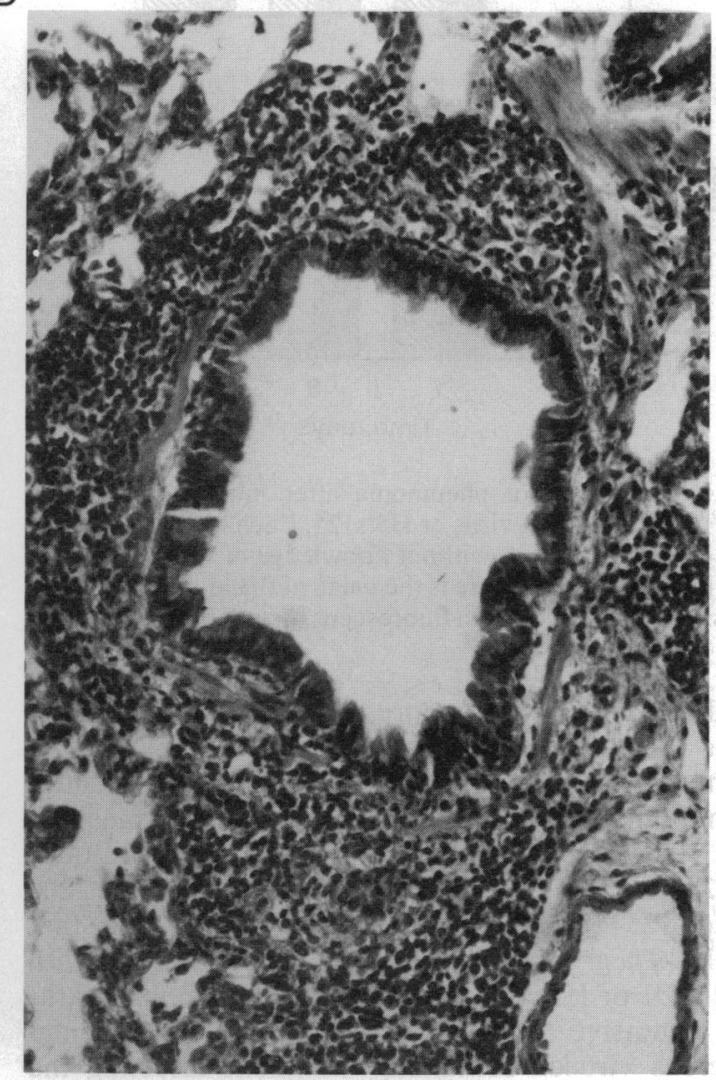

Fig. 2. Pathology of lungs from mice 5 days after intranasal infection with $1 \times 10^{9.0}$ pfu of Ad5 wt virus or H5ts125. (A and $\left.C\right)$ Alveolar infiltration of Ad5 wt and H5ts125, respectively. $(B$ and $D)$ Peribronchial infiltration produced by Ad5 wt and H5ts125, respectively. $(\times 490$.)

While these preliminary experiments to determine the role of $E 1 B$ genes in the mechanism of pathogenesis were in progress, parallel experiments showed that early region 3 $(E 3)$, which is not required for viral replication $(25,26)$, plays 


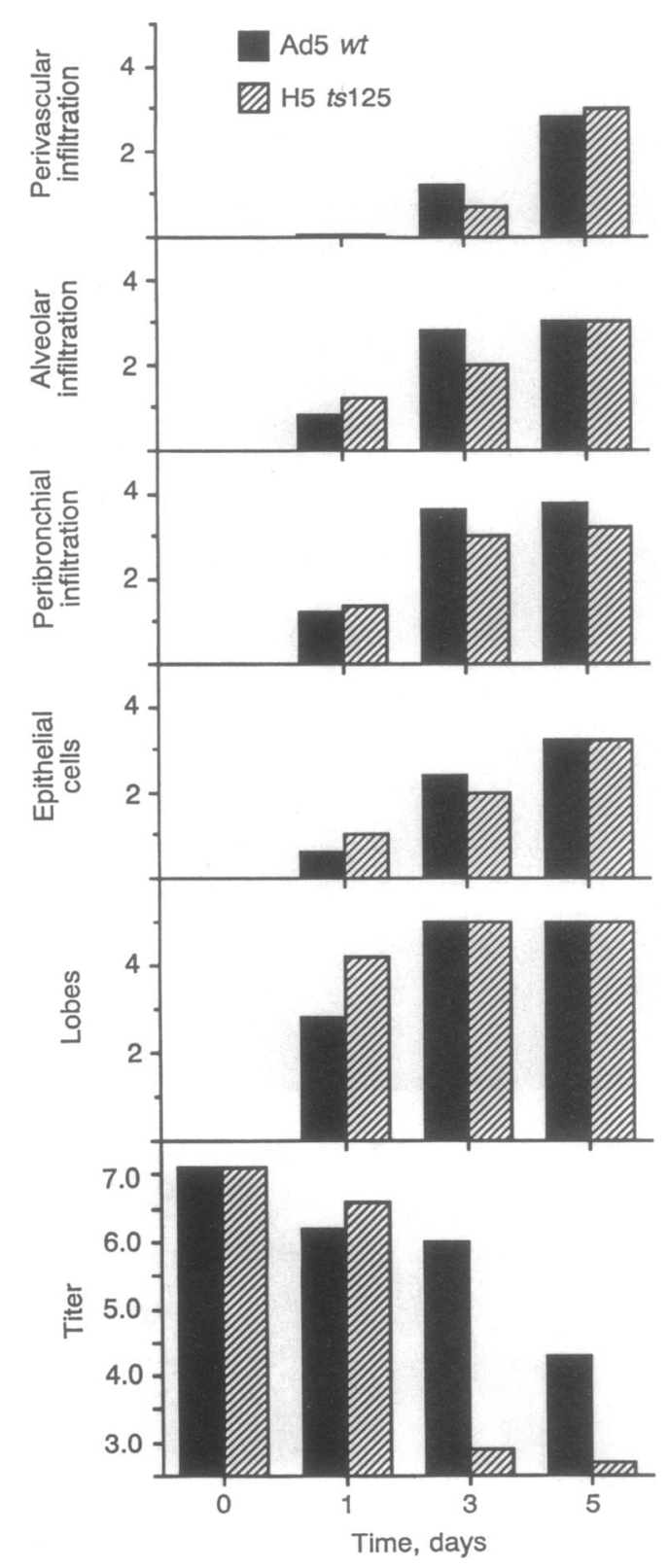

FIG. 3. Development of pneumonia after intranasal infection with $1 \times 10^{9.0} \mathrm{pfu}$ of Ad5 wt virus or H5ts125. Each histological slide was scored on a scale of 0-4 without knowledge of virus employed or day after infection. Each score is the mean of that determined with lungs from six animals. Titer is in fluorescent focus-forming units per gram.

a profound role in actively suppressing the pathogenesis of pneumonia (17). Studies were done with a series of E3 mutants that contain either deletions of almost the entire region or mutations in each of the open reading frames (27) within E3 [e.g., H2dl1801 has a deletion of nucleotides 747-2684 (28) and H5/2d1754 is deleted from nucleotide 1200 to nucleotide 1254 (29), which deletes only the sequence that encodes a 19-kDa glycoprotein]. Infection of cotton rats with either $\mathrm{H} 2 \mathrm{dl} 1801$ or $\mathrm{H} 5 / 2 \mathrm{dl} 754$ produces a pneumonia markedly more extensive than that produced by their parental virus, whereas viruses containing mutations affecting the genes encoding the other $E 3$ gene products do not produce disease different from wt virus (17). The finding that the 19-kDa glycoprotein reduces transport of the class I major histocompatibility complex antigens to the surfaces of infected cells $(17,30-32)$ and that the mutants employed (H2dl1801, H5/2d1754, and others) permitted the class I antigens to be transported to the infected cell surfaces and expressed to the same extent as on uninfected cells, suggested that a cytotoxic $\mathrm{T}$-cell response to infected cells probably plays an important role in the pathogenesis of the pneumonia (17). This conclusion suggested the possibility that the E1B 58-kDa protein may be one of the antigens expressed on the surface of infected cells to serve as a signal for the specific cytotoxic $T$ cells to recognize the infected cells as being foreign. If this possibility is correct, the absence of the 58-kDa protein in H5dl110-infected cells would account for the reduced pneumonia produced by this $E 1 B$ mutant.

The so-called $E 3 B$ region also appears to contain a gene encoding another suppressive gene product. Thus, H5sub304 , which contains an insertion of a small piece of foreign DNA and a deletion from 83.2 to 85.1 map units (28), does not produce a pneumonia that is more extensive than that of Ad5 wt, but the pulmonary infiltration contains many small foci of polymorphonuclear leukocytes (PMNs), which is not seen in wt virus-infected lungs (ref. 17 and unpublished results). These foci of PMNs do not have the characteristics of a bacterial infection but appear similar to those produced by the cachectin/tumor necrosis factor (TNF) (33). Moreover, the region of the $E 3 B$ genome deleted in $\mathbf{H} 5$ sub304 contains a gene encoding a 14.7-kDa protein, which has been shown in vitro to protect adenovirus-infected cells from lysis by TNF (34). Although the pneumonia produced by H5sub304 contains numerous foci of PMNs, which are not seen in Ad5 wt-infected lungs, wt virus-induced pneumonia does contain PMNs scattered within the alveolar infiltration (unpublished data), suggesting that TNF and possibly other cytokines may play an important role in the pathogenesis of adenovirus pneumonia, at least in this animal model.

The gene functions encoded in early region $1 \mathrm{~A}(E I A)$ and early region $4(E 4)$ should also be investigated. Infections with viruses containing mutations located in the unique region of the E1A 13S mRNA, which encodes a 46-amino acid peptide, do not induce any pathology (unpublished data). These results are difficult to interpret, however, since this region of the 289-amino acid E1A protein enhances transcription from all of the other early genes. Therefore, it is impossible to determine whether the affected $E I A$-encoded gene product is required for pathogenesis or fails to produce pneumonia because one or more other early gene products are not efficiently produced. Mutants containing defects affecting only the $E 4$ gene products have not yet been studied.

The results reported above lead to the conclusion that only early gene functions are responsible for the molecular reactions that induce the pathogenesis of adenovirus pneumonia in this cotton rat model. The results of studies using $E 3$ mutants suggested the hypotheses that the early gene products responsible for this pathogenic process induce two reactions: $(i)$ Infected cells induce a virus-specific cytotoxic $\mathrm{T}$-cell response, and one or more early gene products are expressed on the infected cell surfaces to permit them to be recognized by the specific cytotoxic $T$ lymphocytes (the E1B $58-\mathrm{kDa}$ protein may be one such early viral protein). (ii) The early gene products produced in infected bronchial and bronchiolar epithelial cells (and perhaps others, such as macrophages) send out signals that result in the elaboration and release of cytokines, such as TNF, interleukin 1, and interleukin 6.

We are grateful to Ulla Lundholm-Beauchamp and Wai Wong for technical assistance. This research was supported by National Institutes of Health Grant AI12052 (H.S.G.)

1. Rowe, W. P., Huebner, R. J., Gilmore, R. J., Parrott, R. N. \& Ward, T. G. (1953) Proc. Soc. Exp. Biol. Med. 84, 570-573. 
2. Hilleman, M. R. \& Werner, J. R. (1954) Proc. Soc. Exp. Biol. Med. 85, 183-188.

3. Ginsberg, H. S., ed. (1984) The Adenoviruses (Plenum, New York).

4. Ginsberg, H. S. \& Dixon, M. K. (1961) J. Exp. Med. 113, 283-299.

5. Ginsberg, H. S., Bello, L. J. \& Levine, A. J. (1967) in Molecular Biology of Viruses, eds. Colter, J. S. \& Paranchych, W. (Academic, New York), pp. 547-572.

6. Bello, L. J. \& Ginsberg, H. S. (1967) J. Virol. 1, 843-850

7. Bello, L. J. \& Ginsberg, H. S. (1969) J. Virol. 3, 106-113.

8. Ginsberg, H. S. \& Dixon, M. K. (1959) J. Exp. Med. 109, 407-422.

9. Boyer, G. S., Leuchtenberger, C. \& Ginsberg, H. S. (1957) J. Exp. Med. 105, 195-216.

10. Boyer, G. S., Denny, F. W., Jr., \& Ginsberg, H. S. (1959) J. Exp. Med. 110, 827-844.

11. Boyer, G. S., Denny, F. W., Jr., Miller, I. \& Ginsberg, H. S. (1960) J. Exp. Med. 112, 865-882.

12. Levine, A. J. \& Ginsberg, H. S. (1967) J. Virol. 1, 747-757.

13. Levine, A. J. \& Ginsberg, H. S. (1968) J. Virol. 2, 430-439.

14. Ensinger, M. J. \& Ginsberg, H. S. (1972) J. Virol. 10, 328-339.

15. Ginsberg, H. S., Ensinger, M. G. J., Kauffman, R. S., Mayer, A. J. \& Lundholm, U. (1974) Cold Spring Harbor Symp. Quant. Biol. 39, 419-426.

16. Pacini, D. L., Dubovi, E. J. \& Clyde, W. A., Jr. (1984) J. Infect. Dis. 150, 92-97.

17. Ginsberg, H. S., Lundholm-Beauchamp, U., Horswood, R. L., Pernis, B., Wold, W. S. M., Chanock, R. M. \& Prince,
G. A. (1989) Proc. Natl. Acad. Sci. USA 86, 3823-3827.

18. Thiel, J. F. \& Smith, K. O. (1967) Proc. Soc. Exp. Biol. Med. 125, 564-579.

19. Lawrence, W. C. \& Ginsberg, H. S. (1967) J. Virol. 1, 851-867.

20. Flanagan, J. F. \& Ginsberg, H. S. (1962) J. Exp. Med. 116, 141-157.

21. Galos, R. S., Williams, J., Binger, M. H. \& Flint, S. J. (1979) Cell 17, 945-956.

22. Chee-Sheung, C. C. \& Ginsberg, H. S. (1982) J. Virol. 42, 932-950.

23. Babiss, L. E. \& Ginsberg, H. S. (1984) J. Virol. 50, 202-212.

24. Babiss, L. E., Ginsberg, H. S. \& Darnell, J. E., Jr. (1985) Mol. Cell. Biol. 5, 2552-2558.

25. Kelly, T. J. \& Lewis, A. M., Jr. (1973) J. Virol. 12, 643-652.

26. Jones, N. \& Shenk, T. (1978) Cell 13, 181-188.

27. Wold, W. S. M., Deutscher, S. L., Takemori, N., Bhat, B. M. \& Magie, S. C. (1986) Virology 148, 168-180.

28. Challberg, S. S. \& Ketner, G. (1981) Virology 114, 196-209.

29. Bhat, B. M., Brady, H. A., Pursley, M. H. \& Wold, W. S. M. (1987) J. Mol. Biol. 190, 543-557.

30. Burgert, H. G. \& Kvist, S. (1985) Cell 41, 987-997.

31. Anderson, M., Paabo, S., Nilsson, T. \& Peterson, P. A. (1985) Cell 43, 215-222.

32. Severinsson, L. \& Peterson, P. A. (1985) J. Cell. Biol. 101, 540-547.

33. Beutler, B. \& Cerami, A. (1988) Annu. Rev. Biochem. 57, 505-518.

34. Gooding, L. R., Elmore, L. W., Tollefson, A. E., Brady, H. A. \& Wold, W. S. M. (1988) Cell 53, 341-346. 